\title{
Technologies to Characterize Nanostructured Particles and Bulk Materials
}

\author{
Heiner Jaksch, ${ }^{*}$ M. Steigerwald, ${ }^{*}$ V. Drexel* \\ * Carl Zeiss SMT-Nano Technology System Div., Carl-Zeiss-Str. 56, 73447 Oberkochen, Germany
}

Elastic and inelastic scattering at single atoms and excitation of electrons in solids are the fundamental processes that influence the range of electrons, the depth distribution of ionisation and the emission of SE and BSE electrons. New technologies are introduced to separate the different information coming from different scattering processes and hence better characterize nanostructured materials.

Due to the GEMINI principle ${ }^{1,2,3}$, the information, coming from the different scattering processes, is separated and projected via the GEMINI lens to different detectors (SE and EsB) on the beam axis. The signal is directly detected without any losses from conversion plates. This principle enables the detection of very low Z-contrast differences, far below from a standard BSE detector. Latest tests revealed a detection limit of ppm range dopants in low $\mathrm{Z}$ material (Boron in Al2O3). Even different polymers can be differentiated with this technology, due to the fact, that the signal coming from the sample, is amplified by a beam booster. The principle here is Energy selected BSE detection $(E \mathrm{sB})$.

New improvements come from an integrated GEMINI lens detector, selecting and separating the BSE signal via $Z$ contrast and angle contrast. While the high angle BSE electrons, which are detected in the unique in column EsB detector, where the electrons are separated via the Energy, the large and very large angles, coming from different scattering processes are collected in the AsB detector, or Angular selective BSE detection system.

In homogene crystalline bulk materials like metals, or ceramics, we have strong demands to characterize this material and determine the treatment history (cooling, walking, strain). Chemically etching the polished surface is a common technology to describe grain structure (Fig. 3). Sub grain information is lost due to damage. The channelling contrast, coming from mainly Mott scattered electrons, highlights this mechanism, which is used in this detector. The GEMINI lens separates the single scattered electrons from large angle multiple scattered BSE electrons. As a result we detect unmatched crystalline contrast, where normally nothing is visible (Fig 1,2,4). The change in the lattice orientation in some samples is typically less than 0.5 degrees in sub-grains, which is difficult to be detected with EBSD technique (Fig. 1, 4).

Similar to our general GEMINI detection technology - fishing the true (interesting) signal and suppressing the noise - we separate the different scattered electrons in the multimode STEM detection system. Elastic (BF) and inelastic (DF) scattered electrons are separated and detected as usual. Beyond this, we image large angle scattered (LAADF) electrons and look for the orientation of crystals. Similar to the TEM imaging of a series of reflexes, different orientations, strain or defects are visible at a glance. We can detect features, absolutely not visible in normal BF or DF. The interference of Bloch waves in stressed crystals give information about growth stress or doping stress in nano particles (Fig.5, 6). Altogether six basic imaging modes are used in this detection system, which is an extremely useful technology to characterize nano-structured materials.

References

[1] Jaksch H. et. al. Microsc. Microanal. (2004) 1372 CD

[2] Jaksch H. et. al. Microsc. Microanal. 9 (Suppl) (2003) 106

[3] Jaksch H. Field emission SEM for true surface imaging and analysis, Materials World, Oct. 1996 


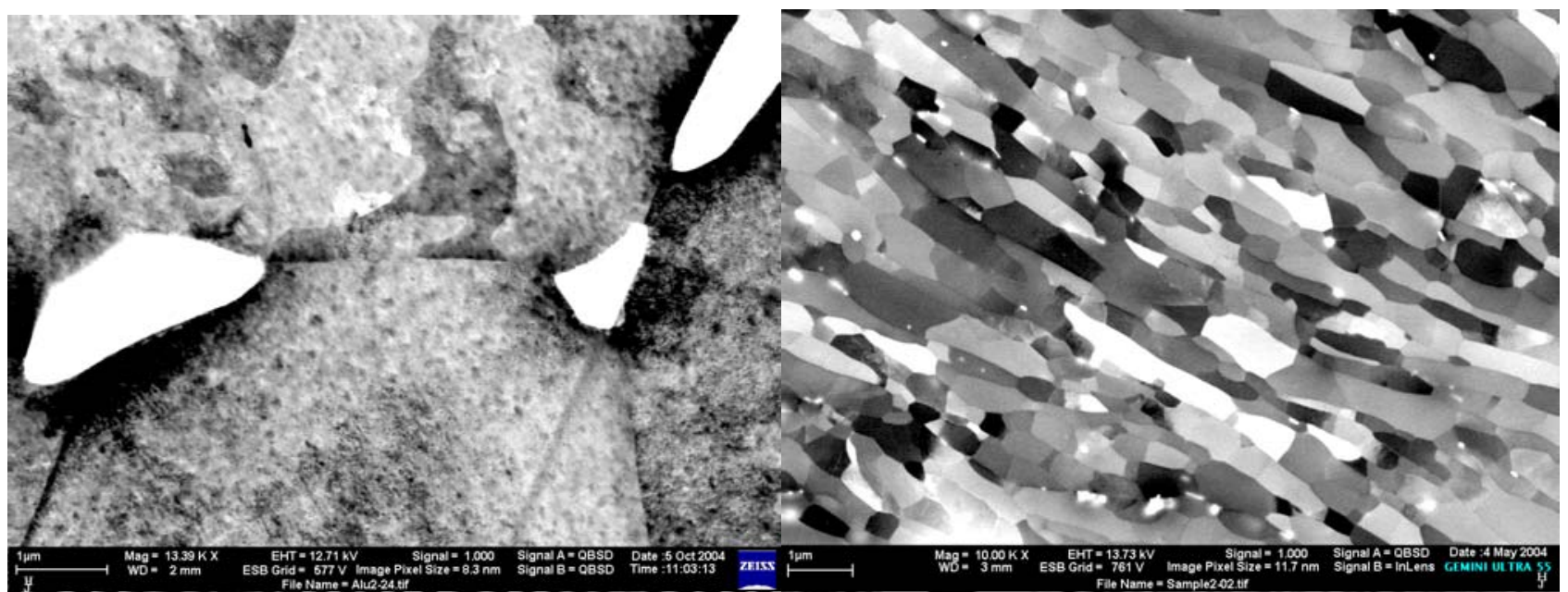

Fig 1:Subgrains and sharp grain boundary in Alu Fig 2:AsB channeling contrast in pure Aluminum

Polished but unetched surface. AsB detector image Only polished but unetched surface.

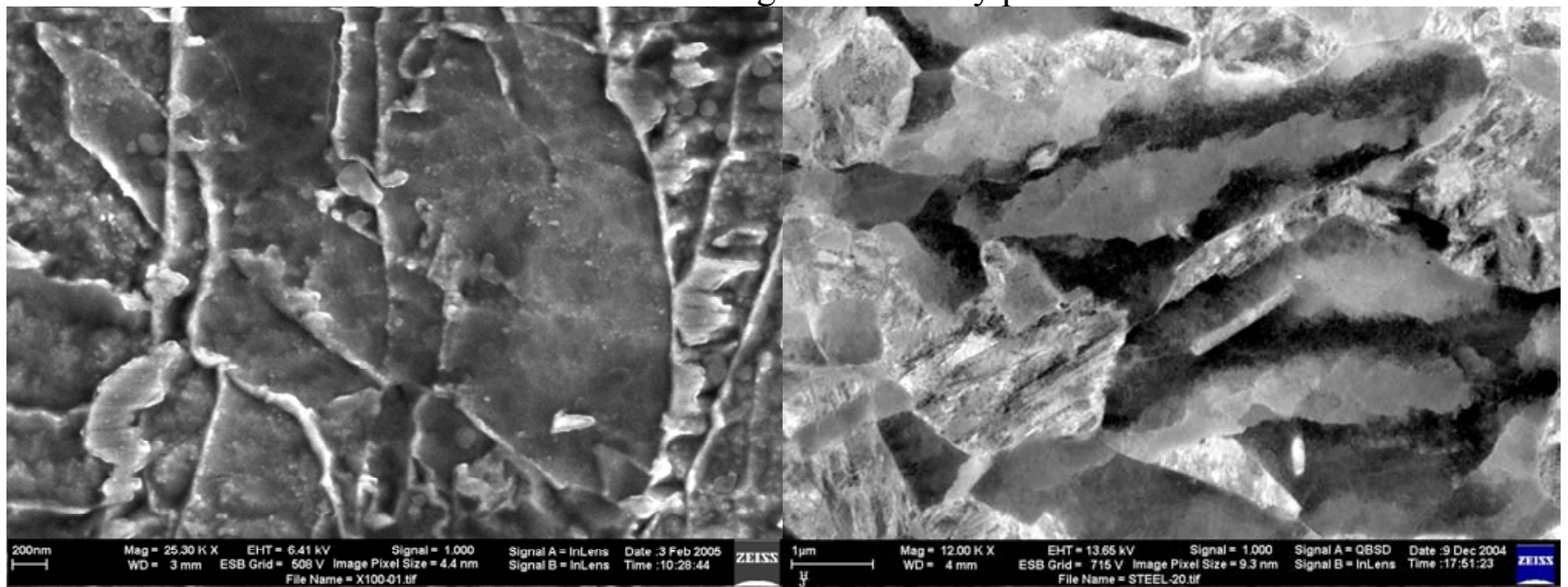

Fig.3:Classical etched steel with grain boundaries Fig. 4: AsB image of steel showing finest grain

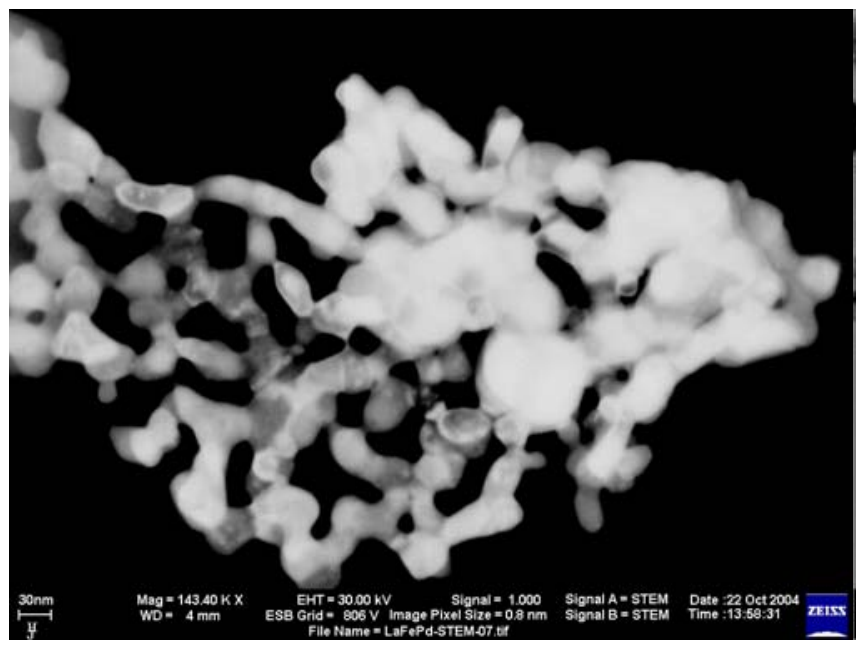

Fig. 5: Classical DF image of LaFePd-Oxide. and subgrain information. Only fine polished.

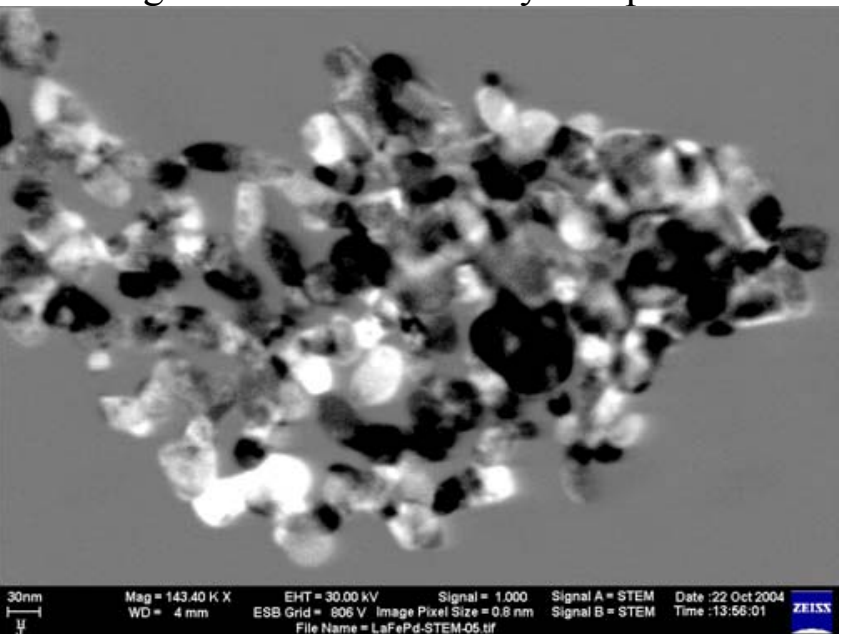

Fig. 6: The same sample clearly characterized in the orientation DF mode of the STEM system. 